Michael B. Frank

\title{
Sanierung und Neukonzeption bestehender Bausubstanz
}

\author{
Das Beispiel der Universitätsbibliothek Konstanz
}

\section{Einleitung}

Die Universitätsbibliothek Konstanz wurde nicht als repräsentatives, freistehendes Gebäude (Tempel der Weisheit) geplant, sondern erstreckt sich als Herz der Universität über mehrere Gebäudeabschnitte der als Universitätsneugründung in den 1960er Jahren geplanten Campus-Universität. Sie ist über fünf Geschossebenen mit den jeweils fingerartig angrenzenden Fachbereichen eng verwoben und über ca. 40 individuelle Zugänge mit ihnen verbunden. Konzeptionell und bautypologisch stellt sie damit einen Sonderfall dar und ist ein herausragendes bauliches Abbild der Humboldt'schen Idee der Universität als integrales Gebäude der Durchdringung von Leben und Lernen.

Die klare und nüchterne, von den in einem sog. Schwarzbuch von 1969 festgehaltenen Baunormen und der Bautechnik bestimmte strukturelle Konzeption des Universitätsgebäudes ist typisch für ihre Entstehungszeit in den 1960er und 1970er Jahren und kann als Musterbeispiel des funktionalen sog. Architektonischen Strukturalismus gelten. Die Universität Konstanz steht daher als Gesamtensemble unter Denkmalschutz.

Die im Folgenden beschriebene Bauaufgabe bestand darin, den neuen Anforderungen an Bibliotheksbauten einerseits und der räumlich gestalterischen und technischen Einbindung in dieses Bestandsgebäude und seine Strukturen andererseits gerecht zu werden.

\section{Gebäudebestand und Aufgabenstellung}

\subsection{Gebäudestruktur}

Die Gebäude der Hochschule wurden als Stahlbetonskelettkonstruktion aus FertigteilKassettendecken gebaut. Die Decken sind im Rastermaß von 7,20 m zwischen Betonunterzüge eingespannt. Nur der etwa 2,1 Mio. Medien umfassende Bibliotheksbestand erhielt eine davon unabhängige Konstruktion, da die Raumanforderungen der Bibliothek nicht mit den Gebäudeparametern (Konstruktionsachsen und Geschosshöhen) übereinstimmten. Die Medienbestände wurden daher in hochregalartigen, begehbaren 
Stahlregalen untergebracht, die über Treppenanlagen von der Primärkonstruktion aus erreichbar sind. Diese begehbaren Türme des Wissens stehen in großzügigen Lufträumen innerhalb der beiden umzubauenden Medienbereiche. Im Rahmen der Sanierung und Neugestaltung wurden etwa $18500 \mathrm{~m}^{2}$ Nutzfläche asbestsaniert und umgestaltet.

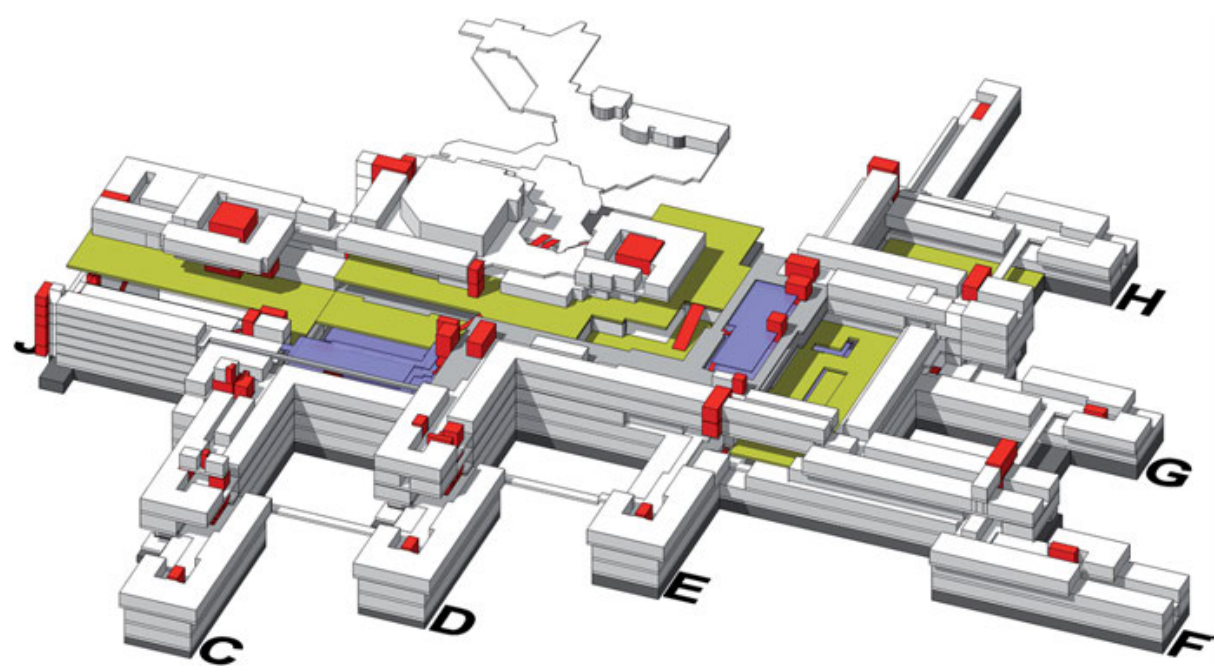

Abb. 1: 3D - Modell der Buchbereiche $S$ und $G$ mit den angrenzenden Fachbereichen (C) Ernst ${ }^{2}$ Architekten AG / M. B. Frank Freier Architekt.

Der zentrale Zugangsbereich für die drei Hauptbuchbereiche (S, J und G) mit etwa $4500 \mathrm{~m}^{2}$ Nutzfläche, das sogenannte Informationszentrum, befindet sich als eigenständiger Gebäudeteil außerhalb der eigentlichen Buchbereiche. Da er den heutigen technisch-funktionalen und räumlich-gestalterischen Anforderungen nicht mehr gerecht wurde, sollte er komplett umgebaut und an die Standards einer modernen digitalen Bibliothek des 21. Jahrhunderts angepasst werden.

In diesem Rahmen wurden gemeinsam mit der Bibliothek moderne Arbeitsund Studierzonen entwickelt, die einerseits den spezifischen Anforderungen einer Wissenschaftlichen Bibliothek entgegenkommen, andererseits aber flexibel genug sind, um individuellen Nutzungsspielraum zuzulassen. Hierzu zählen u. a. neu geschaffene Computerarbeitsräume (CIP-Pools), Computerforschungslabors, Gruppenarbeitsräume, ein anspruchsvoll gestalteter Lesesaal und neugestaltete Einzelarbeitsplätze. Vergleichbare Raumangebote waren bislang im Gebäudebestand nicht vorhanden. ${ }^{1}$

1 Bei der planerischen und baulichen Umsetzung des Bauwerks unterstützte maßgeblich das Planungsteam (s. Factsheet) und insbesondere Nikolaos Dimou von Ernst ${ }^{2}$ Architekten AG. 


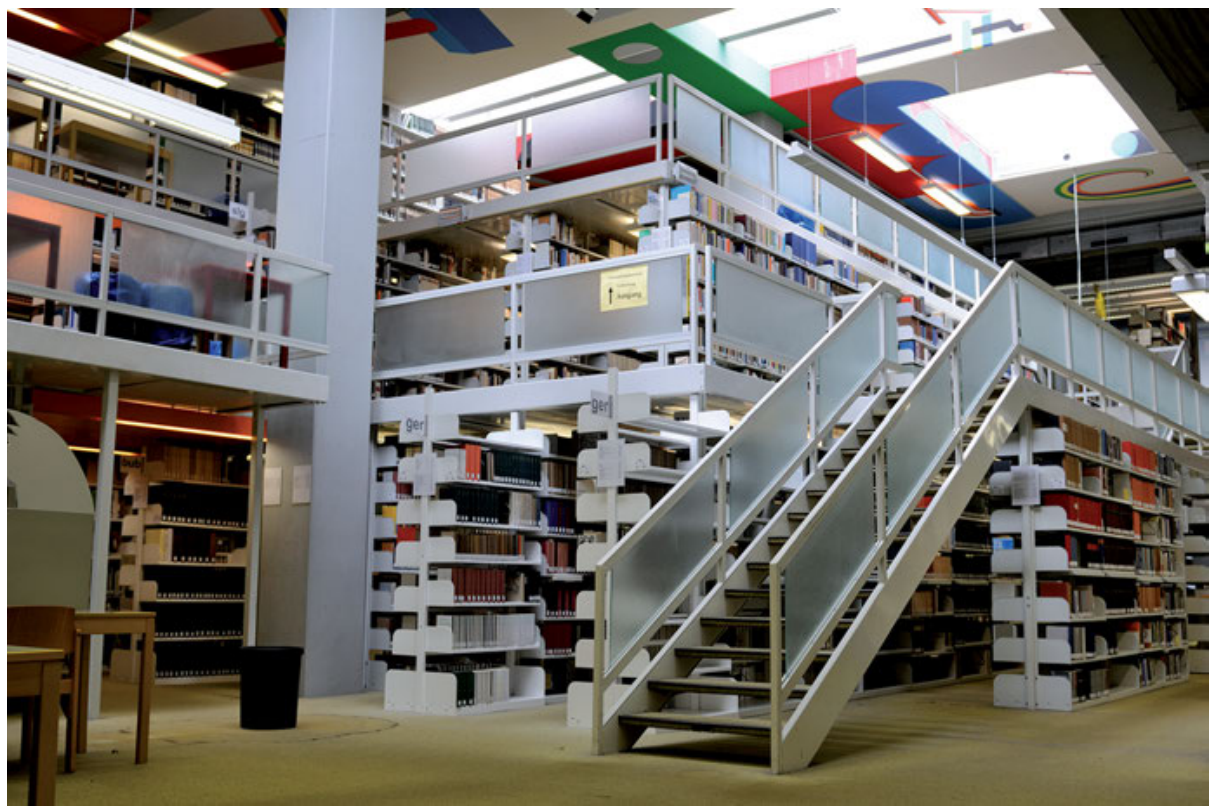

Abb. 2: Bestandssituation Buchbereich G vor dem Umbau (C Universität Konstanz.

\subsection{Asbestsanierung}

Aufgrund von Asbestfunden musste die Bibliothek im Oktober 2010 geschlossen und als asbestverunreinigter Bereich von der allgemeinen Nutzung ausgenommen werden. Eine umfassende Asbestsanierung nach der TRGS 519 war für eine erneute Freigabe der Bibliothek unumgänglich.

Es ist davon auszugehen, dass in Zukunft vergleichbare Hochschulgebäude aus derselben Entstehungszeit ebenfalls vor der Aufgabe der Sanierung und Neugestaltung stehen werden. ${ }^{2}$ Insofern stellt die Schadstoffsanierung der Universitätsbibliothek Konstanz ein geeignetes Fallbeispiel für vergleichbare, noch anstehende Sanierungen dieser Gebäudetypologie dar.

2 Ein berühmtes Beispiel deutscher Universitätsbauten der 1970er Jahre wurde in den 2000er Jahren durch Foster + Partners saniert, die sog. „Rostlaube“ der Freien Universität Berlin (Architekten Georges Candilis, Alexis Josec u. Shandrach Woods, Fassadengestaltung von Jean Prouvé). 


\section{Sanierungsumfang}

Folgende Bauteile wurden auf ihr Gefährdungspotenzial hin untersucht und, soweit erforderlich, durch ein ausgewiesenes und spezialisiertes Sanierungsunternehmen ausgebaut und fachgerecht entsorgt:

- Asbesthaltige Trennwände

Die als räumliche Abgrenzungen zu den Fachbereichen in der Bibliothek eingebauten asbesthaltigen Systemtrennwände (Typ: Mechel-Wände) stellten aufgrund ihres Konstruktionsprinzips ein latentes Gefährdungspotenzial dar, da die Anschlussdetails und die Wandelementstöße damals mit sogenannten Asbestschnüren ausgeführt wurden.

Die asbesthaltigen Trennwände wurden von Gutachtern als Hauptverursacher für die aufgetretene Asbeststaubbelastung in der Bibliothek ausgemacht. Die Asbestfasern wurden v. a. durch die vielen kleinen Beschädigungen in den Wänden, die u. a. durch Nachinstallationen in der über 40jährigen Nutzungsdauer entstanden waren, freigesetzt.

Unabhängig von der baurechtlichen Bewertung mussten daher alle asbesthaltigen Trennwände dauerhaft und vollständig ausgebaut und entsorgt werden.

- Lüftungskanäle Bibliothek

Die aus den Trennwänden ausgetretenen Asbestfasern verteilten sich durch die Lüftungsanlage in alle betroffenen Gebäudeteile. Daher war es aus hygienischen Gründen und aufgrund der Schadstoffkontamination unvermeidbar, das komplette Kanalnetz zu ersetzen.

- Kontaminierte Wand- und Deckenbekleidungen, abgehängte Decken, Bodenbeläge

Die in der Bibliothek vorhandenen Teppichbodenbeläge waren zusätzlich zur Kontamination durch die langjährige Nutzungsdauer in einem desolaten Zustand. Sie wurden deshalb im Zuge der Asbestsanierung vollständig ausgebaut und entsorgt.

- Technik-Installationen

Im Zuge der Asbestsanierung wurden auch sämtliche Technik-Installationen (v. a. Elektro und Medien) altersbedingt ersetzt, unabhängig von ihrer Schadstoffbelastung.

Auch die in Teilbereichen (bzw. in bestimmten Bauteilen) der Bibliothek entdeckten KMF-, PCB- und schwermetallhaltigen Belastungen wurden im Zuge der Schadstoffsanierung fachgerecht ausgebaut und entsorgt. 


\section{Alle Maßnahmen auf einen Blick}

- Ausbau und Entsorgung aller asbesthaltigen Trennwände, Verkleidungen und Decken

- Ausbau und Entsorgung der Zu- und Abluftkanäle sowie der Brandschutzklappen

- Ausbau und Entsorgung der PCB- und schwermetallhaltigen Alt-Fassade

- Ausbau und Entsorgung aller TGA-Installationen in Abstimmung mit den zuständigen Fachplanern

- Ausbau und Entsorgung aller Oberbodenbeläge und KMF-Verwendungen

- Dekontamination / Reinigung von mobilem Inventar, Mobiliar und Kunstwerken

- Dekontamination / Reinigung der stationären Regalbereiche

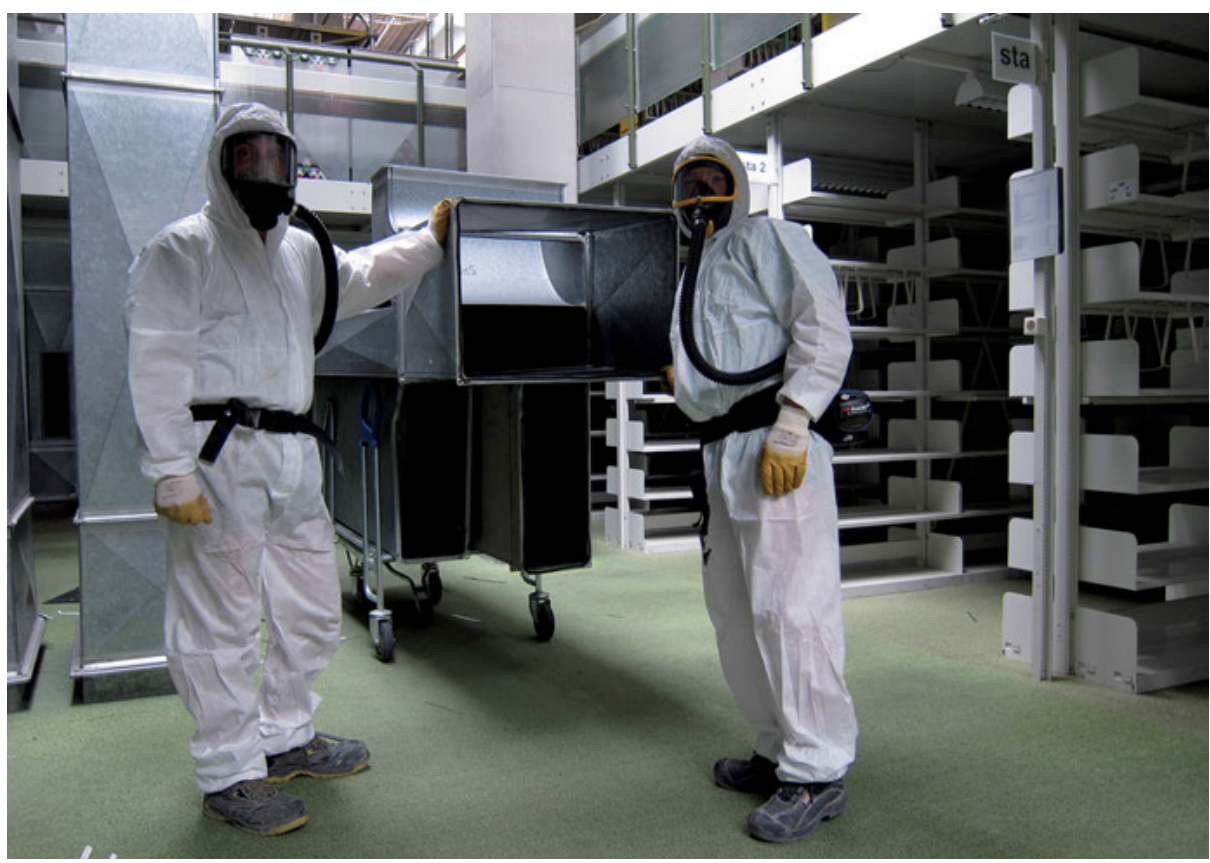

Abb. 3: Asbestentsorgung (C M. Frank. 


\section{Daten und Fakten zur Universitätsbibliothek Konstanz}

Baujahr: 1969/72

Architektur: Universitätsbauamt Konstanz

Sanierung und Neugestaltung:

Bauherr: Vermögen und Bau Baden-Württemberg, vertreten durch das Amt Konstanz

VOF-Verfahren: 2011

Planung und Bau: 2011-2017

Fertigstellung: September 2015

Ernst $^{2}$ Architekten AG in Zusammenarbeit mit Michael B. Frank, Freier Architekt

Gesamtkonzeption und Entwurfsplanung: Michael B. Frank

Ausführungsplanung: Ernst ${ }^{2}$ Architekten AG und Michael B. Frank

Projektteam: Jana Herzog, Christina Schmidinger, Mirko Schnabel

Bauausführung: Ernst ${ }^{2}$ Architekten AG

Projektleitung: Nikolaos Dimou

Geschoss-Ebenen: 5

Buchregal-Ebenen: 6

Medienbestand: ca. 2 Mio. ME

Nutzfläche Buchbereich S: ca. $6900 \mathrm{~m}^{2}$

Nutzfläche Buchbereich G: ca. 7500 m² $^{2}$

Nutzfläche Informationszentrum: ca. $4300 \mathrm{~m}^{2}$

Gesamtnutzfläche: ca. $18700 \mathrm{~m}^{2}$

Gesamtbaukosten: ca. 30 Mio. Euro

Materialien und Hersteller:

\begin{tabular}{lll} 
Böden & Carpet Concept & http://www.carpet-concept.de \\
\multirow{2}{*}{ Wände } & BASF mastertop & http://www.basf.com \\
& Feederle Systemwand & http://www.feederle.de \\
& Strähle Raum-Systeme & http://www.straehle.de \\
& Knauf Trockenbau & http://www.knauf.de \\
& Jansen Brandschutzverglasung & http://www.jansen.com \\
& kvadrat Textilien & http://www.kvadrat.dk \\
Decken & Knauf Trockenbau & http://www.knauf.de \\
& Ecophon Schallabsorber & http://www.ecophon.com \\
& zumtobel leuchten & http://www.zumtobel.com \\
& XAL Leuchten & http://www.xal.com \\
Möbel- & Vitra & http://www.vitra.com \\
ausstattung & Sondermöbel & http://www.mb-frank-architektur.de
\end{tabular}


Nach Abschluss der Entsorgungsarbeiten stellten sich die Buchbereiche in einer puren, strukturalistischen Rohbauästhetik dar, die den Ausgangspunkt für den Neuausbau und die Neugestaltung bildete.

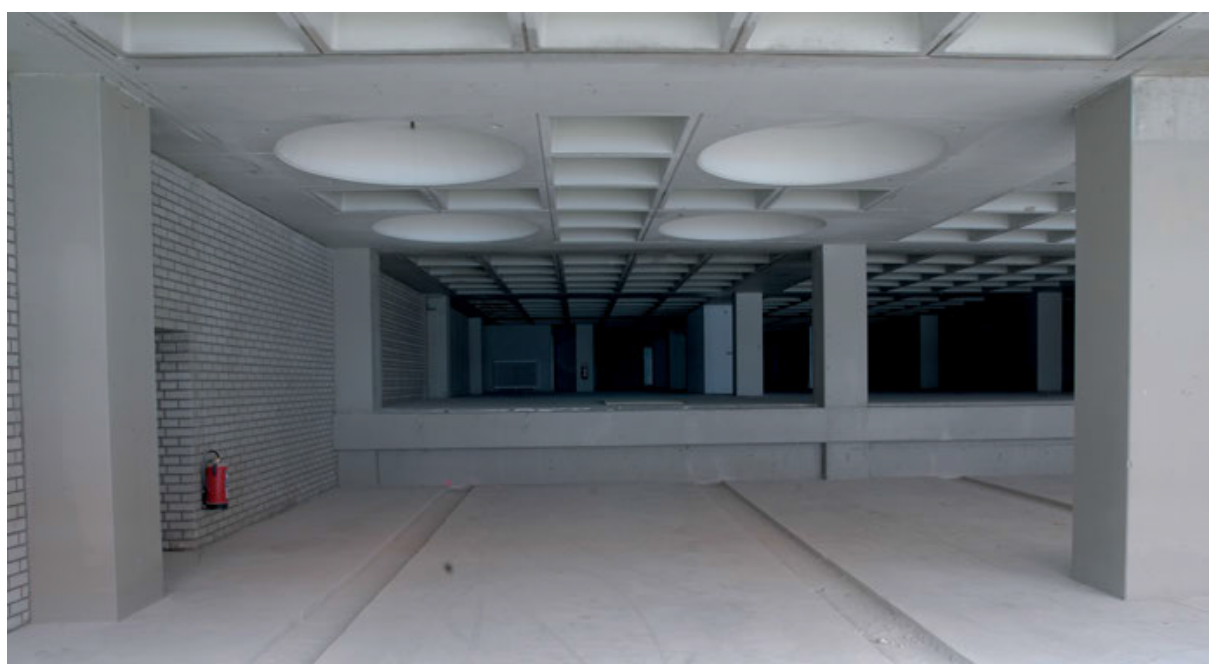

Abb. 4: Zustand nach Rückbau (Betonfertigteilbauweise im Informationszentrum) @ M. Frank.

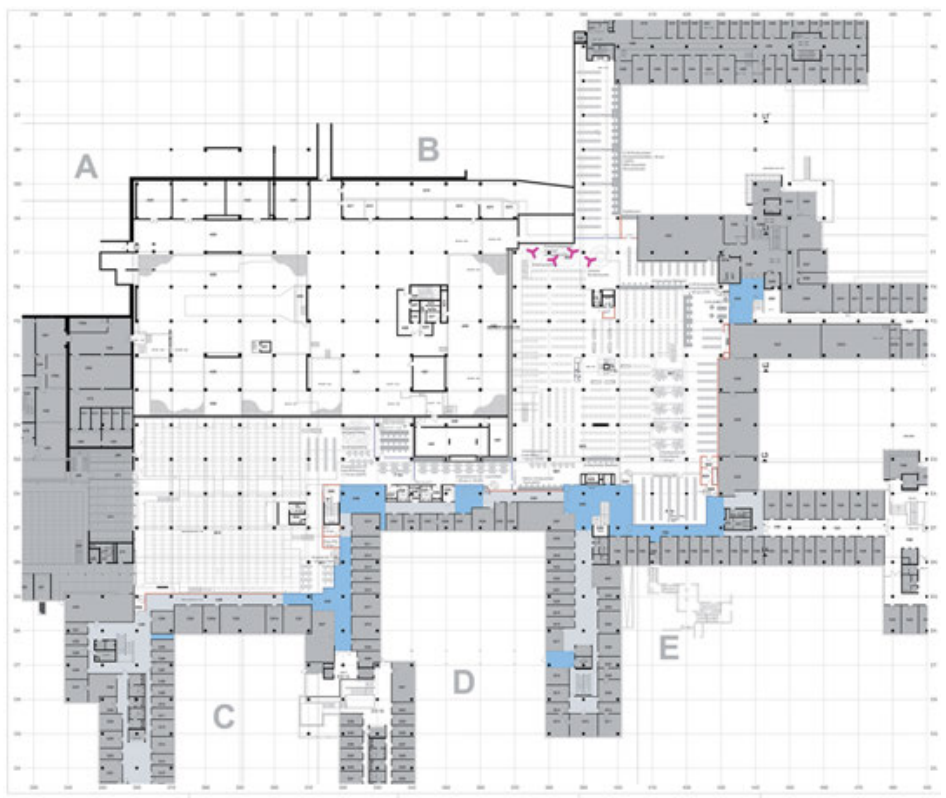

Abb. 5: Grundrissplan von Buchbereich $S$ und $G$ der Bibliothek (unterste Ebene 02 von 6 Ebenen) $(\subset$ Ernst $^{2}$ Architekten AG / M. B. Frank Freier Architekt. 
Die eingangs beschriebene enge Verzahnung der Fachbereiche mit ihren jeweiligen Medienbeständen - damals die Leitidee des Gebäudeentwurfs - führt heute, unter strenger gewordenen baurechtlichen und v. a. brandschutzrechtlichen Rahmenbedingungen, zu einer Reihe planerischer Problemstellungen und schwieriger Detaillösungen. Diese stellten aufgrund der Komplexität der Gesamtanlage eine besondere Herausforderung dar.

Vor allem musste ein nachhaltig funktionierendes, vorbeugendes Brandschutzkonzept erarbeitet werden. Häufig können aus Kostengründen bzw. aus Gründen der Komplexität des Gebäudebestands nicht alle geforderten Brandschutzmaßnahmen im Rahmen einer Baumaßnahme sofort ausgeführt werden. Deshalb wurde im Falle der Sanierung der Universitätsbibliothek Konstanz ein umfassender Maßnahmenkatalog erstellt, in dem festgelegt wurde, welche brandschutztechnischen Umbauten über die kommenden Jahre verteilt realisiert werden müssen. Diese planerische Vorgehensweise ist typisch und übertragbar auf die brandschutztechnische Ertüchtigung komplexer Bestandsarchitektur dieser Baujahre.

\section{Urkonzeption und Zeitgeist}

Die Gesamtanlage der Universität Konstanz wurde in den 1960er Jahren durch das Universitätsbauamt Konstanz unter der Leitung von Wenzeslaus Ritter von Mann als Neugründung geplant und im sprichwörtlichen Sinne auf die grüne Wiese des Gießbergs gesetzt. Der Gießberg befindet sich in wunderbarer naturräumlicher Aussichtslage über dem Überlinger See, einige Kilometer außerhalb der Stadt Konstanz.

Die Gebäudeanlage folgt zwar einerseits der damals gültigen Doktrin der Funktionalität und Nüchternheit ${ }^{3}$, die nicht so sehr vom Kontext ausgeht, sondern vorwiegend auf sich selbst Bezug nimmt. Andererseits ist die Universität Konstanz keine reine ,Maschine‘, sondern nimmt in vielfältigen Details Bezug zur umliegenden Landschaft und zur Topografie auf.

Visionäre architektonische Konzepte von Büros wie Archigram aus London oder auch die der sogenannten Pop-Art hatten, wenn auch in abgeschwächter Form, Einfluss auf die konzeptionelle und formale Gestaltung der Hochschule. Die Universität Konstanz ist mit ihrer sowohl konsequenten als auch sehr eigenständigen Interpretation dieser Ideen ein herausragender Vertreter der Architektursprache dieser Zeit des Aufbruchs. Der Gründungsgedanke der Konstanzer Universität war vom Wunsch nach einer universitären Reform getragen. Dieser Reformgedanke hat hier eine einzigartige konzeptionelle und formale Umsetzung in die bauliche Gestalt gefunden.

Die Architektur der Universität Konstanz kann maßgeblich vor diesem kulturgeschichtlichen Hintergrund verstanden werden. Ausgehend von dieser Interpretation

3 Vgl. Naumann 2009. 
dienten einige wesentliche Leitgedanken der Entstehungszeit der Hochschule als Orientierungspunkte für die Form- und Gestaltfindung der Neukonzeption. Auf diese drei Prinzipien des alten und des neuen Gestaltungskonzeptes soll im Folgenden näher eingegangen werden.

\subsection{Raster und Freiheit}

Wie bereits beschrieben ist das Gebäudeensemble der Universität Konstanz ein typischer Vertreter der rationalen, strukturalistischen Architekturauffassung und damit als bauhistorischer Klassiker der 1960er/70er Jahre einzustufen. Das Besondere an der Gestaltungsphilosophie der Universität Konstanz zeigt sich in den vielfältigen Spielarten der sog. Freien Form, die parallel zum nüchternen Rationalismus des (1,20-m-Betonfertigteil-) Rasters und der Bandfassaden das Gebäude durchziehen.

Die Freie Form wird zum einen durch die allgegenwärtige Durchdringung des rauen und nüchternen Bauwerks mit Kunst am Bau erreicht. Zum anderen tragen zahlreiche spielerische Elemente der Freiraum- und Fassaden- bzw. der Bauteilgestaltung zum sehr individuellen Erscheinungsbild der Universität bei. Die undogmatische Leichtigkeit in der Form- und Farbgebung steht im Kontrast zu dem ansonsten funktional geprägten Gebäude. Dieser Kontrast macht das Besondere am architektonischen Ausdruck der Universität Konstanz aus. Nicht zuletzt kann das Spiel von Raster und Freiheit als formaler bzw. architektonischer Ausdruck der inhaltlichen Reformbestrebungen bei der Neugründung der Bodenseeuniversität gesehen und interpretiert werden.

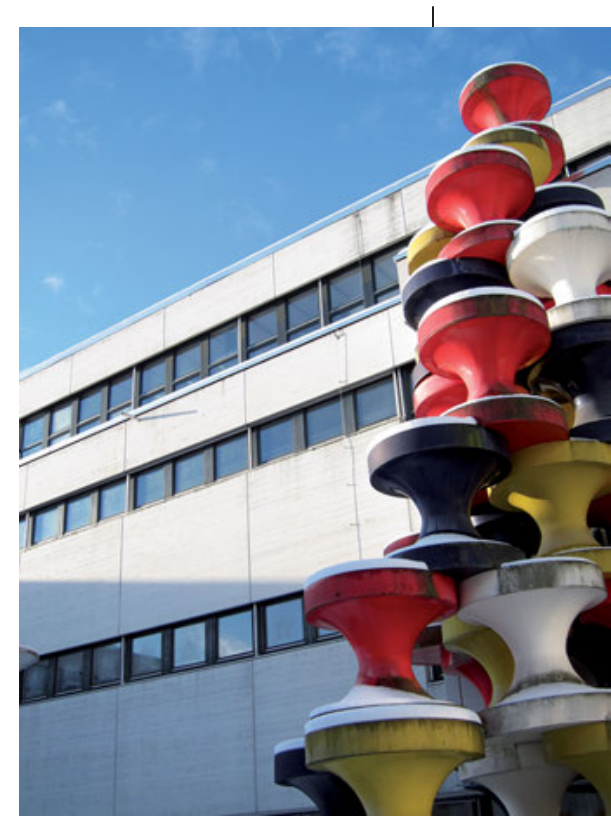

Abb. 6: Spindelturm vor der Bandfassade (C) M. Frank. 
Das kontrastreiche Spiel von Regel und Ausnahme, Klarheit und Mystik, Freiheit und Restriktion steht auch in der Tradition der klassischen Moderne Le Corbusiers. Für die architektonische Neugestaltung der Innenräume - bis hin zur Möbelausstattung - wurde dieses Prinzip hier bewusst aufgegriffen, um den innovativen, reformerischen Geist und die Aufbruchsstimmung, durch die das Gebäude entstanden ist, weiterzuschreiben und dabei bewusst auf die Stärken des ursprünglichen Konzepts zu setzen. Das Gebäude hat, obwohl es 40 Jahre alt ist, immer noch eine frische, ungezwungene Ausstrahlung, die es zu erhalten und fortzuschreiben lohnt. ${ }^{4}$

\subsection{Vielfältige Bezüge zwischen Außenraum und Innenraum}

Aufgrund der oben beschriebenen Gebäudekonzeption mussten erhebliche Bereiche der Bibliothek unterirdisch, d. h. ohne direkten Außenbezug, geplant werden. Dadurch strahlt sie an manchen Stellen den „Charme einer Tiefgarage“ (so eine Besucherin) aus. Daraus leiteten sich für die Neugestaltung zwei Entwurfsprinzipien ab: zum einen ein klares, die Struktur des Gebäudes unterstreichendes Beleuchtungskonzept, zum anderen die konsequente Betonung der bestehenden Sichtbezüge und die Schaffung neuer Durchblicke und Ausblicke nach draußen. Das neue Gestaltungskonzept schafft in dieser Form - in Anlehnung an das ursprüngliche Entwurfsprinzip - vielfältige Bezüge zwischen innen und außen.

Bereits vorhandene bauliche Blick- und Raumbeziehungen wurden aufgegriffen und verstärkt. Dort, wo ein Außenbezug besonders reizvoll oder notwendig erschien, wurde dieser neu geschaffen.

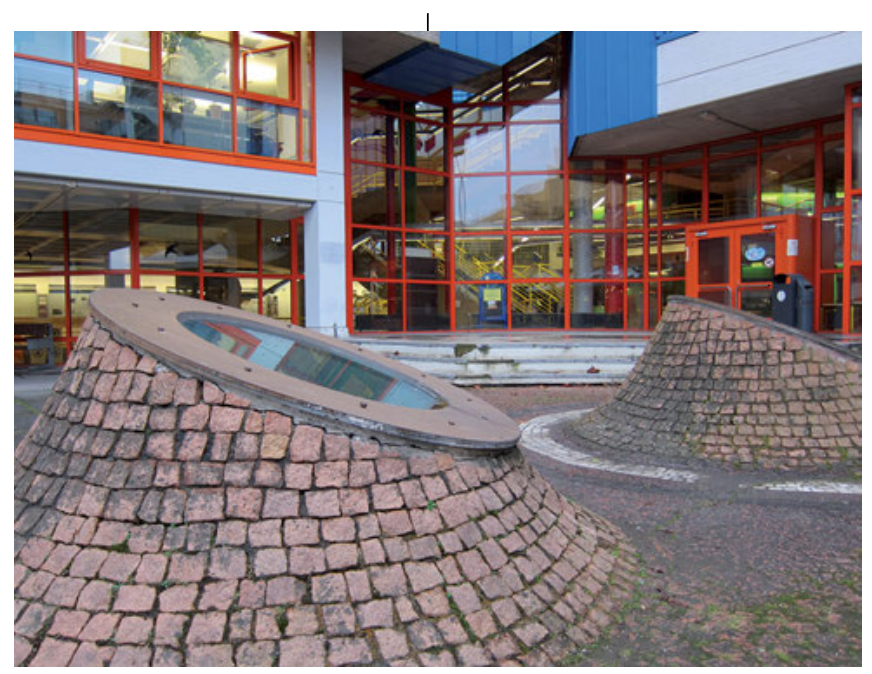

Abb. 7: Bullaugen: Sichtbeziehung nach draußen @ M. Frank.

4 Vgl. in ähnlicher Weise die Pausa-Tonnenhalle: Frank 2011 sowie Henning 2011. 


\subsection{Kommunikation}

Im Rückblick auf die Entstehungszeit der Universität ist das Thema der Kommunikation, im direkten wie im übertragenen Sinne, eines der Hauptanliegen der sogenannten Reformuniversität, die im Zusammenhang der 1968er Studentenbewegungen entstand. Konstanz sollte modern und zeitgemäß sein. Insofern war Kommunikation nicht nur Zeitgeist, sondern auch Programm.

Diesem Anspruch an eine moderne und zeitgemäße Hochschule will und muss Konstanz auch heute im Zusammenhang der Exzellenzinitiative nachkommen.

Die Schaffung eines kommunikativen und lebendigen Lehr- und Lernumfeldes für die heutige Zeit war insofern eine Hauptanforderung an das Neugestaltungskonzept. So gesehen wurde durch die Neugestaltung eine Idee der Gründungszeit - man könnte durchaus auch sagen: deren Geist - aufgegriffen und innerhalb der heutigen Universitätslandschaft weiterentwickelt und variiert.

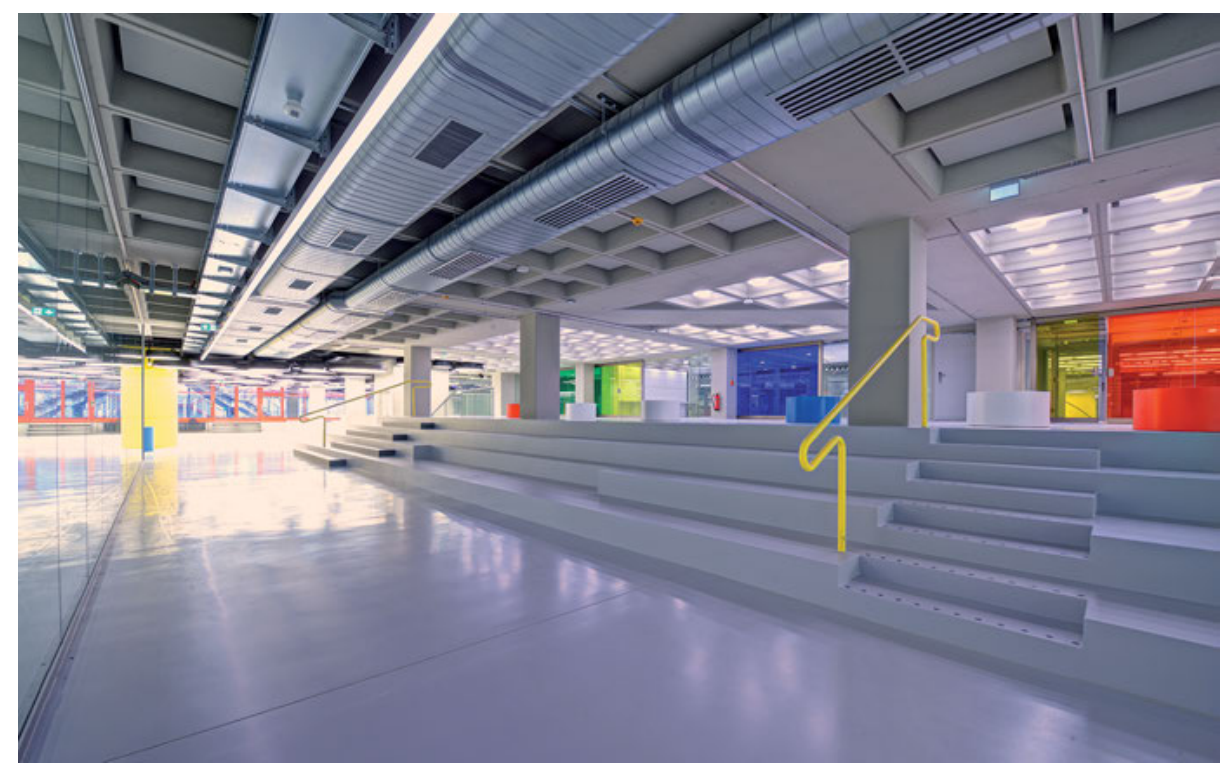

Abb. 8: Sitzstufenlandschaft zum Mediendeck (c) I. Reiter.

Die baulich stark verdichtete Gebäudeanlage ist wie eine enge, kleine Stadt angelegt, mit ausgeklügelten Wegeführungen und vielen informellen Begegnungsstätten. Das Informationszentrum war bisher zwar die zentrale Anlaufstelle. Der Marktplatz der Bibliothek war jedoch nicht als kommunikativer Ort gestaltet. Ziel war es daher, den Zugangsbereich offener und transparenter zu gestalten. Im Rahmen der Neugestaltung sollten im Informationszentrum Räume für neue Nutzungsmöglichkeiten integriert werden. Diese wurden sämtlich als Glasboxen geplant und so im Raum 
angeordnet, dass sie die Wegeführung in die Buchbereiche unterstützen und den Eingangsbereich subtil gliedern und begrenzen. Die neue Sitzstufenlandschaft trägt ebenso zu diesem kommunikativen Ansatz bei, der sich bis in die technischen Details und Feinheiten einer vernetzten, digitalen technischen Ausstattung auf der Höhe der heutigen IT-Technologie fortsetzt. Dadurch erhielt der zentrale Zugangsbereich der Bibliothek Offenheit und Großzügigkeit, ähnlich einer klassischen Agora.

\section{Die konkrete Umsetzung}

\subsection{Gestaltungselemente}

Bei der Sanierung der Universitätsbibliothek Konstanz musste zunächst ein zeitgemäßes Raumprogramm gemeinsam mit der Bibliothek entwickelt und in den bestehenden Räumlichkeiten organisiert werden. Da es sich bei der Bibliothek, wie bereits beschrieben, nicht um ein freistehendes Gebäude handelt, wurden im nächsten Schritt ein klares und prägnantes innenarchitektonisches Farb- und Materialkonzept sowie zahlreiche Ausstattungsdetails erarbeitet.

Die drei beispielhaften Gestaltungselemente, auf die im Folgenden genauer eingegangen werden soll, sind in der Praxis nicht immer klar voneinander abzugrenzen: Innerhalb eines architektonischen Objekts vereinen und überlagern sich in der Regel oftmals zwei oder drei der genannten Elemente. Dennoch soll hier versucht werden, diese aus dem Kontext heraus erarbeiteten, grundlegenden Prinzipien der architektonischen Gestaltfindung als einzelne Themen kurz zu beschreiben.

Für die bauliche Umsetzung vor Ort zeichnete das auf die Baü̈berwachung spezialisierte Architekturbüro Ernst ${ }^{2}$ Architekten AG verantwortlich, das die detailgenaue Umsetzung der gestalterischen Vorgaben in enger Kooperation mit dem Büro des Verfassers realisierte.

\subsubsection{Die Form}

Form und Raum, ihre gegenseitige Durchdringung, ihre Materialität und Proportion, die Atmosphäre, die sie im Miteinander schaffen: All dies sind die Grundlagen jeder architektonischen Gestaltung. Vor dem Hintergrund der oben beschriebenen Ur-Konzeption und des einzigartigen atmosphärischen Umfelds der Universität Konstanz war es wichtig, dem Gebäude durch die neue architektonische Intervention keinen fremden, modernen Duktus ,aufzudrücken', sondern den überlieferten, zeittypischen Stil aufzugreifen, neu zu interpretieren und in den unterschiedlichsten Ausprägungen durchzudeklinieren. In keinem Detail war der Wunsch vorherrschend, 
dem Bestand formal etwas gänzlich Neues, Kontextloses gegenüberzustellen oder gar einen bewussten formalen Kontrast zu provozieren.

Im Gegensatz dazu wurden gestaltprägende Einzelelemente, auch wenn sie formal sehr eigenwillig erschienen oder gar heutigen Anforderungen an Schall- oder Brandschutz nicht mehr genügten (wie z. B. die hölzerne „Himmelstreppe“ im zentralen Luftraum), aufgearbeitet und damit sozusagen als Intarsie erhalten.

Die architektonische Haltung gegenüber dem Bestand war von Respekt geprägt und beschränkte sich bewusst auf die Herausarbeitung der Essenz und der Verstärkung dessen, was bereits da war - im Sinne eines Zitats oder einer Interpretation. Wie bei einem Cover-Song wurde das freie, unkonventionelle Gedankengut der 1960er Jahre als Inspirationsquelle aufgegriffen und deren Essenz, sozusagen die Melodie, mit neuen Mitteln in die heutige Zeit übertragen.

Das Betonfertigteilraster beherrscht und prägt das Gebäude über tausende von Quadratmetern. Demzufolge werden auch zukünftig sämtliche Technikinstallationen (Lüftung, Elektroinstallation, IT) sichtbar unter der Betonkassettendecke geführt, die Lüftung in abgerundeten, formschönen Ovalkanälen, die Elektroinstallationen in offenen Kabelpritschen und langen linearen oder runden, abgependelten Leuchten.

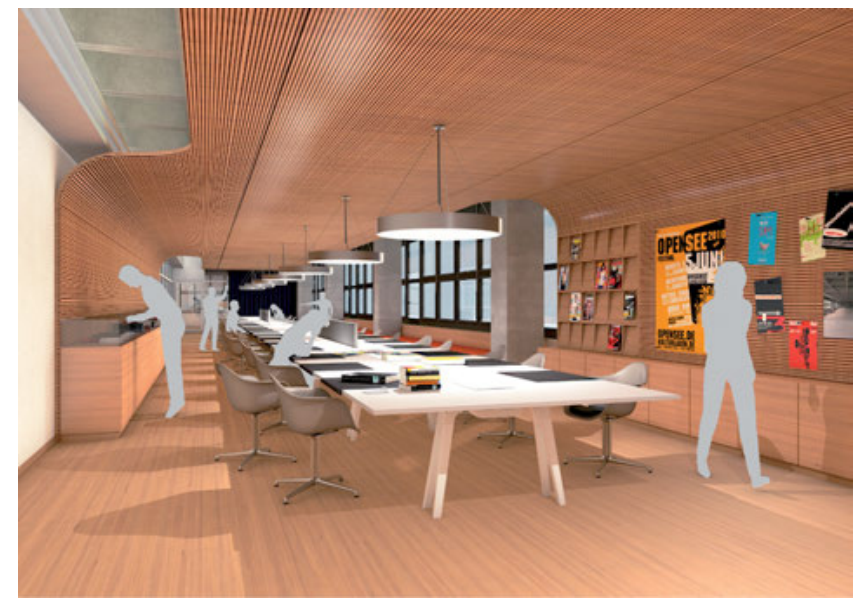

Abb. 9: Perspektive großer Lesesaal (C) Ernst ${ }^{2}$ Architekten AG / M. B. Frank Freier Architekt.

Der gestalterische Reiz entsteht auch hier im Wechselspiel zwischen dem alles beherrschenden Raster und der offenen und freien Form der neu integrierten Elemente. Verbuchungs- und Beratungstheken, die Verkleidungen der Technik, die Beleuchtung oder die frei eingestellten Aufenthalts- und Arbeitsräume (Lesecafé und CIP-Pool, bis hin zur Möblierung) antworten der Eckigkeit und Rechtwinkligkeit der Grundstruktur in Anlehnung an die Urkonzeption mit einer weichen Formensprache - frei nach dem Motto: „Das Runde muss ins Eckige ...“.

Sämtliche neu integrierte Arbeits- und Aufenthaltsräume wurden als transparente, gläserne Boxen, als Raum im Raum gestaltet, wodurch sie der Raumglie- 
derung der Bibliothek, hier insbesondere dem Informationszentrum, eine vorher nicht dagewesene, fließende Großzügigkeit und Transparenz geben und sich zudem elegant der Bestandsstruktur unterordnen.

\subsubsection{Die Farbe}

Wie kaum eine andere Hochschule aus der bundesdeutschen Neugründungsphase Mitte bis Ende der 1960er Jahre ist die Universität Konstanz geprägt von einer freien und frischen, undogmatischen Materialität und Farbgebung - ein bewusster Gegenpol zur nüchternen Struktur des Gebäudes. Gleichzeitig wurde dadurch ein Bezug zur umliegenden Natur und den jungen Studierenden hergestellt.

Für die neue Farb- und Materialkonzeption wurde diese spielerische und undogmatische Haltung in unterschiedlichen Varianten aufgenommen und innerhalb des neuen, zeitgemäßen Raum- und Nutzungskonzepts weitergeführt. An die Stelle der eigenwilligen, für heutige Verhältnisse unkonventionell erscheinenden Farbigkeit der 1970er Jahre tritt eine stärker am Nutzer orientierte Gestaltung mit Farb- und Materialakzenten. Der Grundduktus der neuen Farbigkeit ist von Zurückhaltung geprägt und bewusst ruhig und sachlich im Grundtenor.

Helles Grau ist die vorherrschende Farbe im Informationszentrum und den Wissenstürmen der Buchregale. Diese zurückhaltende Farbwahl ist einerseits eine Referenz an die klare strukturelle Tektonik des Bauwerks, andererseits als neutraler Hintergrund für das bunte Treiben der studentischen Nutzer gedacht.

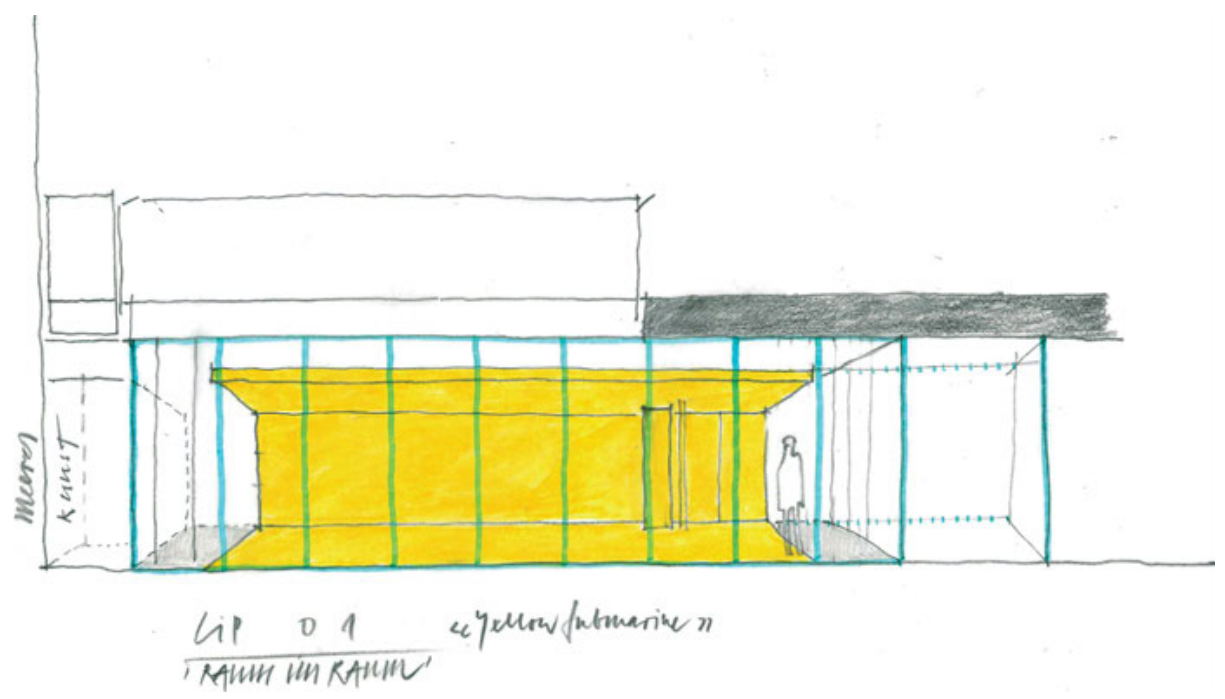

Abb. 10: Skizze „Yellow Submarine“ (c) M. Frank. 
Die neuen Sonderräume (Medienlabors, Bürobox, Lesecafé und CIP-Pools) wurden durch starke Farbakzente hervorgehoben. Dies sind die Bereiche, in denen Austausch und Gespräch stattfinden, wo Neues entstehen kann und soll - wie in einem klassischen Forum.

So entstand zum Beispiel ein gelber Raum - das Yellow Submarine - der gewissermaßen als räumliches Zitat auf die 1960er und 1970er Jahre Bezug nimmt. Neben ihrer ikonografischen Funktion evoziert die Farbe Gelb auch psychologische Komponenten wie Wärme, Helligkeit und Kreativität, wie sie ihr in Goethes Farbenlehre zugeschrieben wurden. Darüber hinaus war die Intention, den Raum mit seiner unmittelbaren Umgebung in Dialog treten zu lassen. Dort, an der tiefsten Stelle der Bibliothek, ist eine bauzeitliche künstlerische Wandgestaltung mit maritimen Motiven aufgebracht. Mit dem „Yellow Submarine“ entstand ein expressiver, stark akzentuierter Raum, der sich dennoch mit der Bestandssituation zu einem neuen Ganzen verbindet.

Was könnte besser zu einem Raum am tiefsten, dunkelsten Punkt der Bibliothek passen, als in dieser Weise die Gedanken anzuregen (und ganz nebenbei auch noch auf die benachbarte maritime Kunst am Bau) zu antworten?

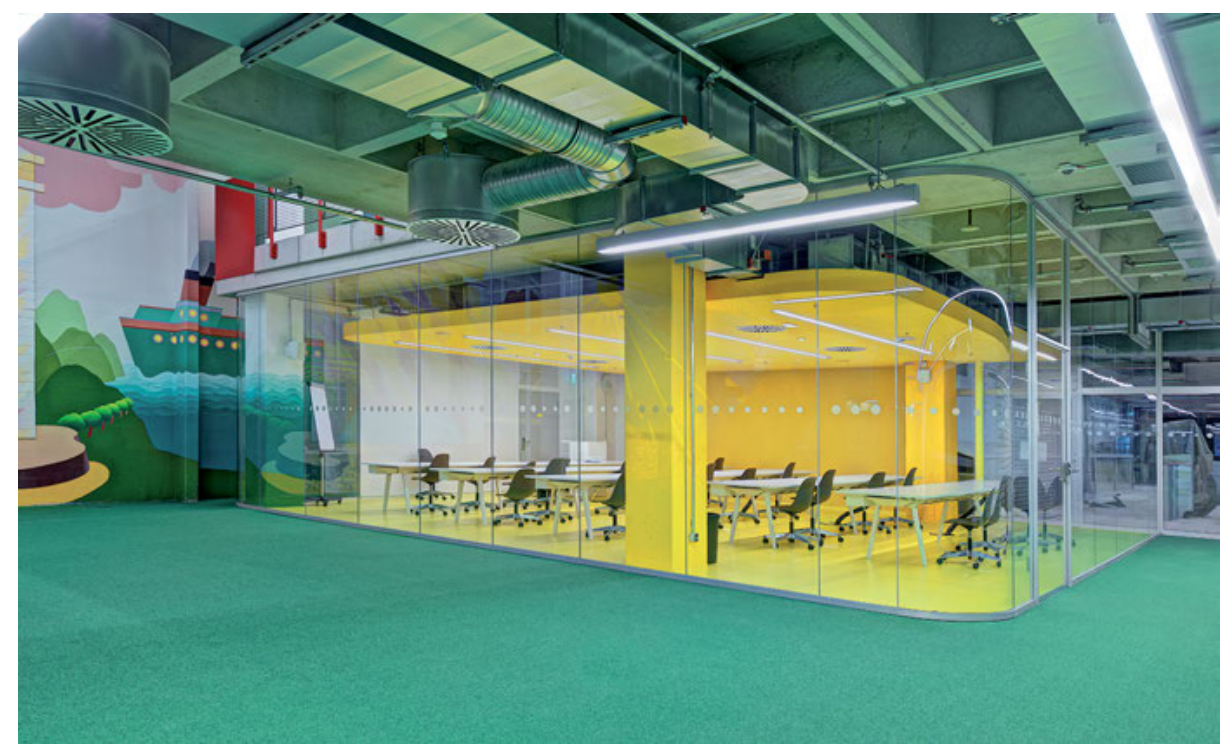

Abb. 11: Computerarbeitsraum und Seminarraum „Yellow Submarine“ (C I. Reiter.

Weitere Farbzitate finden sich in Orange und Weiß und in Form von großflächigen farbigen Glastafeln in den sechs Spektralfarben, die gezielt im Gebäude verteilt wurden. Wie selbstverständlich erleichtern diese farbigen RaumBoxen die Orientierung innerhalb der Bibliothek und unterstreichen jede Ebene in ihrer jeweiligen ikonografischen Farbigkeit. 


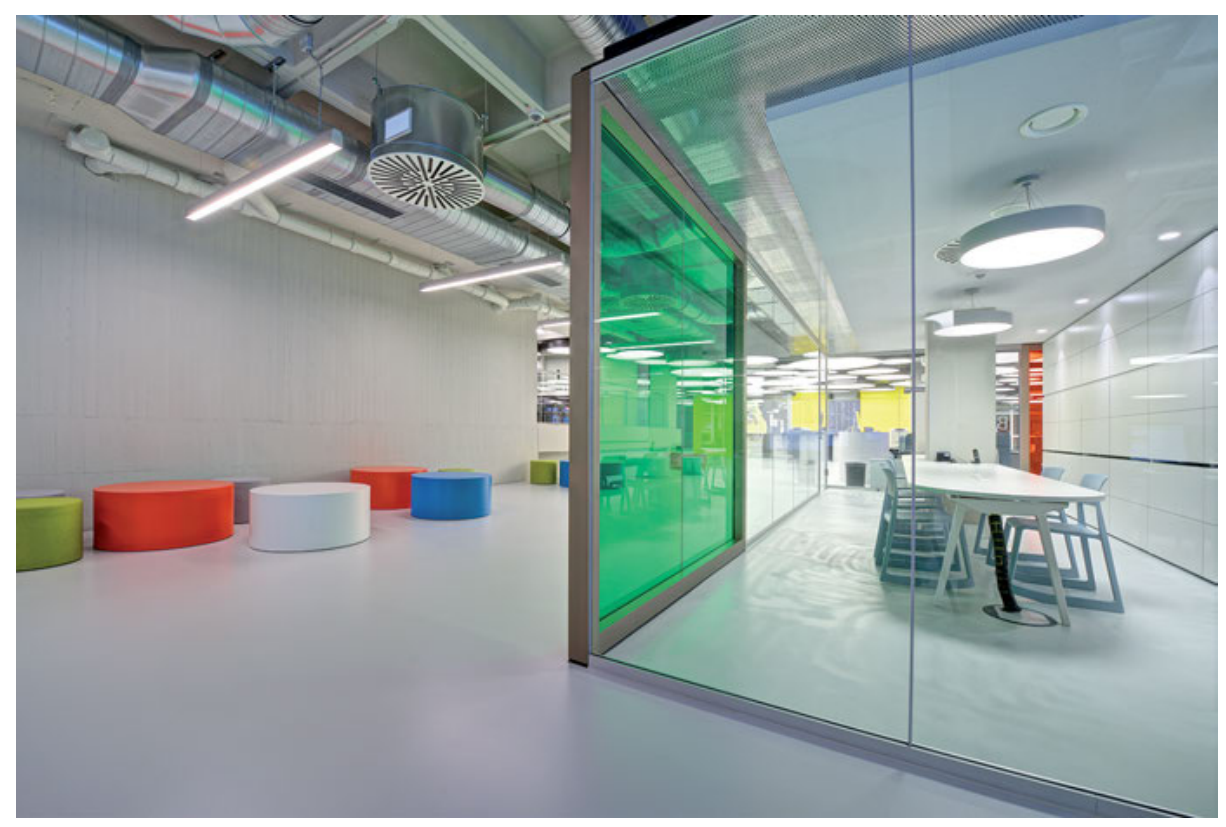

Abb. 12: Durchblick durch farbige Glastafeln (C) I. Reiter.

\subsubsection{Licht und Akustik}

Das Licht, v. a. künstliches Licht und seine Steuerung, spielten bei diesem zum Teil unterirdischen Projekt eine wichtige Rolle. Da die Bibliothek an 350 Tagen im Jahr 24 Stunden geöffnet hat, musste ein technisch und gestalterisch gut durchdachtes, wirtschaftlich tragfähiges Beleuchtungskonzept entworfen werden. Bereits zu Beginn der Entwurfsbearbeitung wurden seitens der Gestaltung klare Leitlinien vorgegeben, die im Laufe der Projektbearbeitung in ständiger Absprache mit den zuständigen Fachingenieuren technisch vertieft wurden.

Die Architekten trugen bereits damals dieser besonderen Situation Rechnung, indem sie an den wenigen baulich umsetzbaren Stellen aufwendige Oberlichtkonstruktionen einbauten, um so den beschriebenen Bezug zur Außenwelt herzustellen. Dadurch konnte jedoch nur in recht bescheidenem Umfang natürliches Tageslicht in die ausgedehnten Räumlichkeiten der Bibliothek gebracht werden.

Neben einer flächendeckenden und ausreichend starken Beleuchtung für Ganztagesarbeitsplätze sowie einer optimalen Ausleuchtung der engen Buchregaltürme (auf Wunsch der Bibliothek sollten alle Regalfachböden optimal (> 500 lx) ausgeleuchtet werden!) war in den stärker frequentierten Bereichen der Bibliothek zusätzlich eine qualifizierte Raumakustik sicherzustellen. 
Die Raumakustik war in den vergangenen 40 Jahren in der Universitätsbibliothek Konstanz nicht beachtet worden. Es existierten im Bestand keine schallabsorbierenden Oberflächen. Dementsprechend wurde von Seiten der Bibliotheksleitung mit Nachdruck eine Verbesserung der Raumakustik (und damit der Arbeits- und Studienbedingungen) durch einen verbesserten Schallschutz eingefordert. Dieser Anforderung wurde aufgrund des baulichen Bestands (Betonkassettendecken) sowie der vielen Technikinstallationen unter der Decke mit speziell für dieses Bauvorhaben entwickelten Licht-Akustik-Bauelementen nachgekommen. Auf diese speziell für den Ausbau in Konstanz entwickelten Bauteile, die Licht und Akustik kombinieren, wird noch detailliert einzugehen sein.

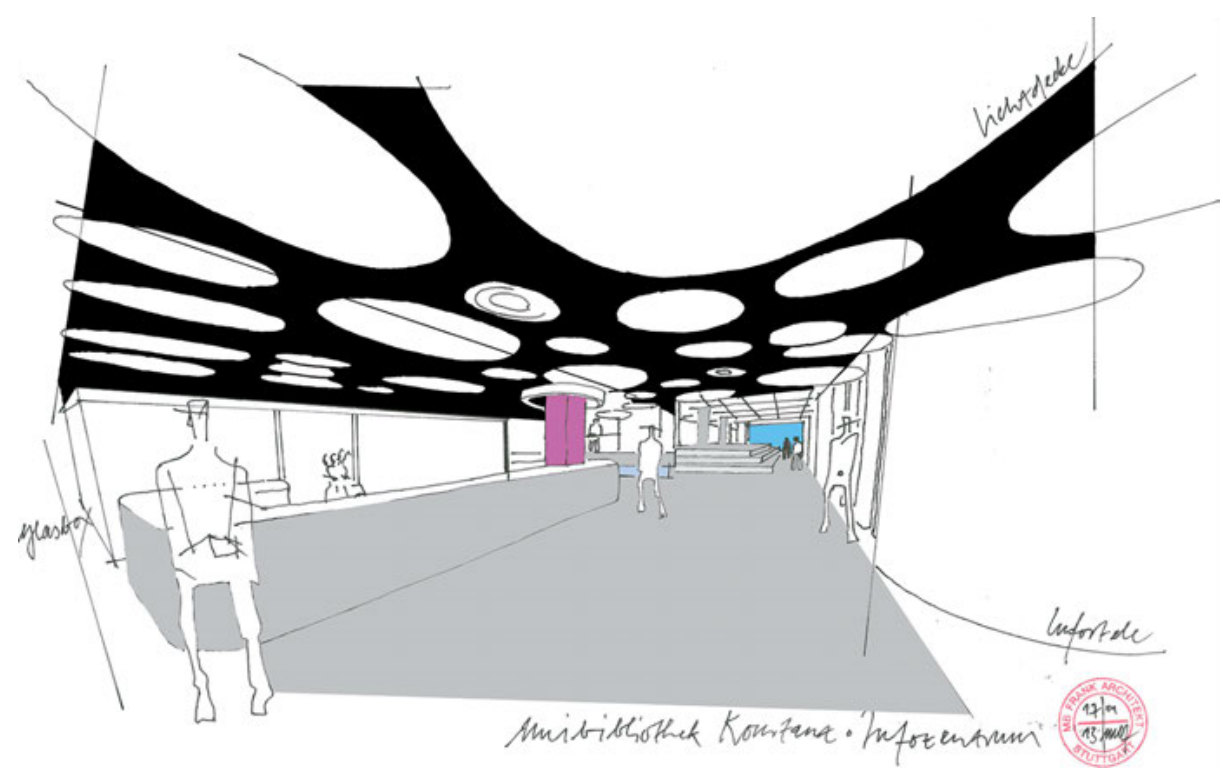

Abb. 13: Skizze Wolkendecke () M. Frank.

\subsection{Bauteile}

Die Buchbereiche S und G sind seit ihrer Erstellung Ende der 1960er Jahre baulich kaum verändert und auch nicht an die heutigen Anforderungen einer modernen Wissenschaftlichen Bibliothek, z. B. mit unterschiedlich gestalteten Arbeitsbereichen, angepasst worden. Somit herrschte vor dem Umbau ein regelrechter Sanierungsstau im technischen wie im programmatischen Sinne. Durch mehrere für die besondere Situation in Konstanz entwickelte Maßnahmen konnten die vorhandenen Defizite, v. a. in den Bereichen der Raumakustik, der Beleuchtung, der Orientierung und der Raumaufteilung, behoben werden. 


\subsubsection{Boden - Teppich und Polyurethan}

Der Boden ist die größte raumakustisch wirksame Fläche in der Universitätsbibliothek Konstanz. Die Decke konnte, wie oben beschrieben, aufgrund der umfangreichen, sichtbar montierten Technikinstallationen (Lüftungskanäle, Elektrotrassen, Beleuchtung), wegen der meist geringen Raumhöhe und nicht zuletzt auch aus Kostengründen nur in Teilbereichen akustisch wirksam verkleidet werden.

Es musste daher ein geeignetes Produkt gefunden werden, welches die geforderte raumakustische Verbesserung bewirken, gleichzeitig hohe Robustheit aufweisen und nicht zuletzt auch konzeptionell und formal zum prägenden Gesamterscheinungsbild des 1970er-Jahre-Bauwerks passen würde. Bei Bemusterungen mit allen Beteiligten wurden sämtliche Aspekte gründlich abgewogen. Am Ende wurde aus einer großen Palette dasjenige Produkt ${ }^{5}$ ausgewählt, welches durch seine grobe, offene Webstruktur und durch einen zusätzlichen filzartigen Rücken (Unterseite des Teppichs) hohe schallabsorbierende Eigenschaften mit einer robusten Sisaloptik verbindet, die formal sehr schön in den Gesamtkontext passt.

Außer den technischen Anforderungen kam dem Farbkonzept eine wichtige Bedeutung zu. Die gezielte Farbwahl ergab sich aus den oben beschriebenen konzeptionellen Rahmenbedingungen des Projekts. Der Boden der untersten Bibliotheksebene und der angrenzenden Fachbereiche ist in Analogie zur grünen Wiese des Gießbergs in kräftigem Grün ausgeführt (Bezug zwischen Innenraum und Außenraum, s. o.). In den weißgrauen, begehbaren Bücherregalen wurde Ton in Ton ein hellgrauer Boden verlegt, wodurch der solitäre Charakter der Wissenstürme gestärkt wird.

Diese gegenüber der Urkonzeption differenzierter ausgeführte Farb- und Materialverwendung stärkt die Klarheit des Raumkonzepts. Sie verbessert die Orientierung, schafft eine klare Zonierung und dient so der funktionalen Gliederung der Bibliotheksbereiche.

Die nutzerspezifischen Anforderungen an die Ausführung des Bodenbelags im Informationszentrum waren ebenfalls Robustheit, Widerstandsfähigkeit und Pflegeleichtigkeit. Der textile Bodenbelag hatte sich während der langjährigen Nutzungsdauer wegen der starken Frequentierung des zentralen Zugangsbereichs nicht bewährt. Der in den öffentlichen Bereichen der Hochschule großflächig verlegte, bauzeittypische Noppenboden schied aus, da das Bewegen der Bücherwagen auf diesem unebenen Bodenbelag zu einer zu großen Geräuschentwicklung führen würde. Die Wahl fiel auf einen Bodenbelag, der sprichwörtlich hart im Nehmen ist und durch seine durchgehend homogene Oberfläche die neue Großzügigkeit des Informationszentrums verstärkt. ${ }^{6}$ Die Bodenbeschichtung auf der Basis von lösungsmittelfreiem

5 Eco Tec von Carpet Concept. http://www.carpet-concept.de/produkte/carpets/flachweben/eco/ eco-tec.html (18.10.2015).

6 Mastertop von BASF.http://www.master-builders-solutions.basf.de/de-de/produkte/mastertop/215 (18.10.2015). 
Polyurethan ist langlebig und kann bei Bedarf auch ausgebessert und nachbearbeitet werden. Der Vorteil gegenüber Parkett- oder anderen harten und robusten Bodenbelägen ist der deutlich bessere Trittschalldämmwert. Durch die flüssige Einbringung, ähnlich einer Estrichüberdeckung, ist diese Beschichtung außerdem in der Lage, vorhandene Bodenunebenheiten und schlechten Estrichzustand auszugleichen.

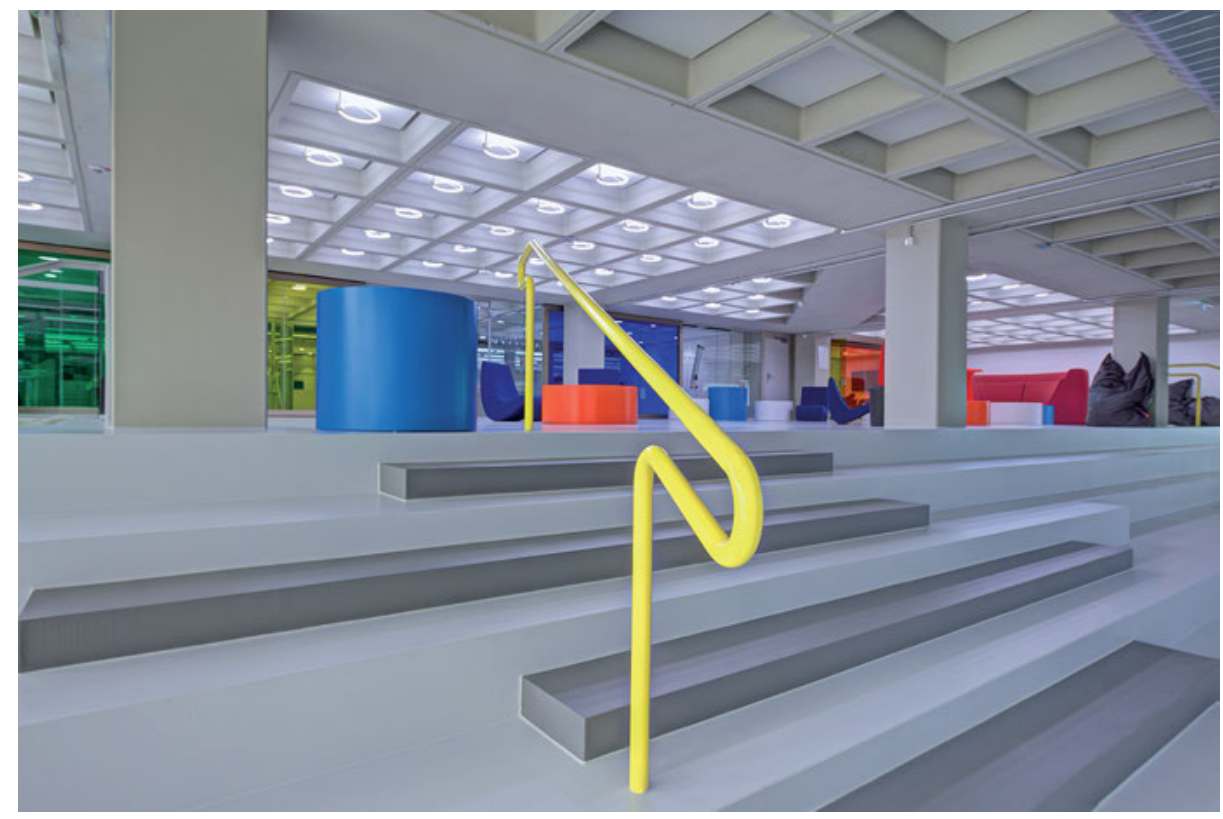

Abb. 14: Informationszentrum nach der Fertigstellung @ I. Reiter.

Da der harte Bodenbelag in den bauphysikalischen Rechenmodellen raumakustisch nicht angerechnet werden konnte, musste eine verbesserte Schallabsorption im Informationszentrum durch zusätzliche Maßnahmen erreicht werden. Auf diese soll in den beiden folgenden Abschnitten näher eingegangen werden.

\subsubsection{Licht-Akustik-Decke}

Wie bereits beschrieben, kam in Konstanz der Einbau einer klassischen Schallschutzdecke (z. B. Gipskartondecke) nicht in Frage. Um eine bessere Raumakustik zu erreichen, musste eine Deckenlösung entwickelt werden, die beides leistet: Schallabsorption und eine gleichmäßige, großflächige Ausleuchtung.

Die für die Konstanzer Situation entwickelte Lösung besteht aus 133 runden Einzelleuchten, die unterseitig mit einer mikroperforierten, lichtdurchlässigen Spezialfolie bespannt sind. Diese Sonderanfertigung wurde in drei unterschiedlichen 
Durchmessern von $80 \mathrm{~cm}, 120 \mathrm{~cm}$ und $200 \mathrm{~cm}$ so unter die Decke und zwischen die Technikinstallationen gehängt, dass sie die hohe Installationsdichte überspielt und gleichzeitig immer eine maximale Raumhöhe erzielt wird. Durch die spielerische Anordnung (Raster und Freiheit, s. o.) der Akustikleuchten entsteht eine unverwechselbare Raumwirkung, die den Hauptzugang betont und der Bibliothek ein starkes Image verleiht. Das dadurch entstehende Bild erinnert an die Leichtigkeit eines Wolkenhimmels und spielt damit auch auf das Konzept von Innen und Außen an.

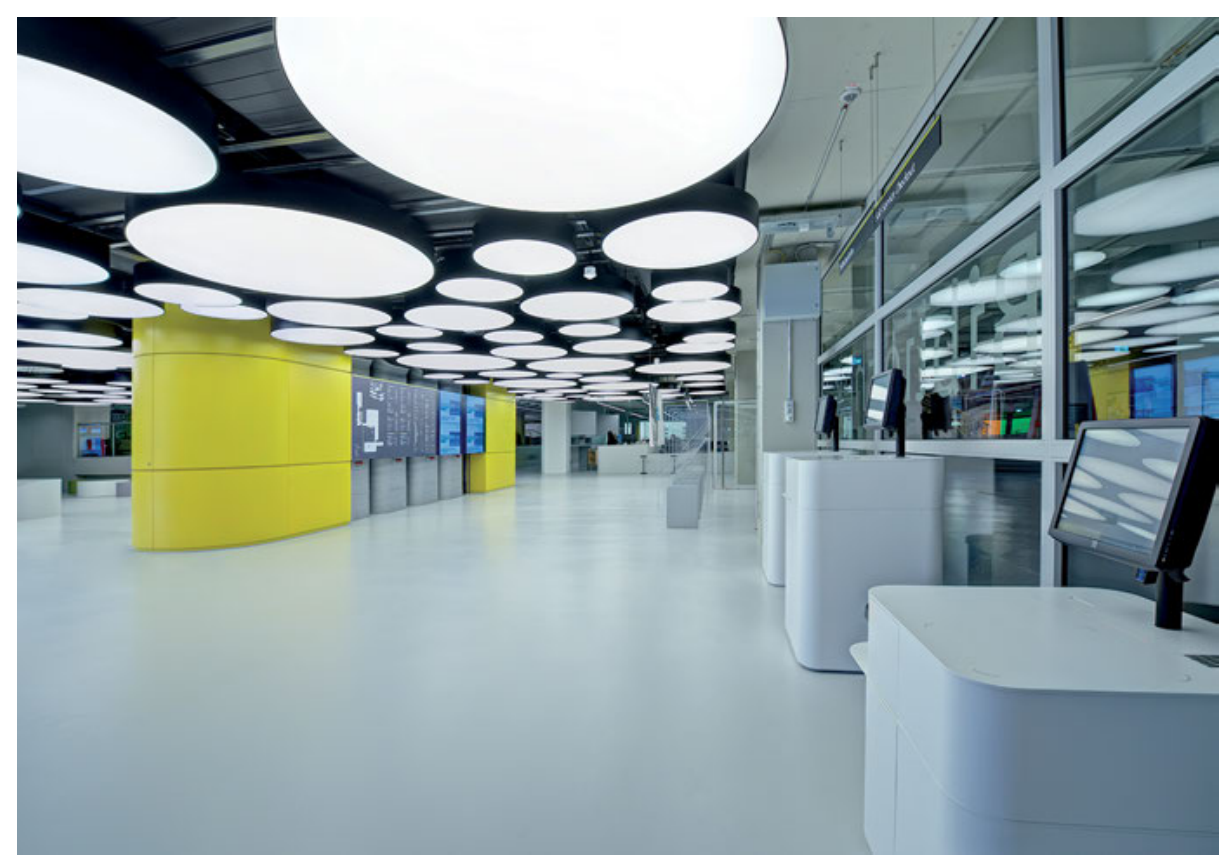

Abb. 15: Informationszentrum, Wolkendecke im Eingangsbereich @ I. Reiter.

\subsubsection{Licht-Akustik-Wand}

Da sich die raumakustische Wirkung der Licht-Akustik-Decke nur auf den ca. $500 \mathrm{~m}^{2}$ großen Eingangsbereich erstreckt, waren für die anderen Arbeits- und Aufenthaltsbereiche des Informationszentrums weitere Maßnahmen zu treffen.

Zunächst wurden über die gesamte Fläche der Arbeits- und Aufenthaltsräume innerhalb der Betonkassetten der Deckenkonstruktion Schallabsorber eingebaut. Diese schufen die Basis für die Raumakustik.

Eine weitere Besonderheit der akustischen Maßnahmen - neben der Licht-AkustikDecke - ist die über 50 m lange Lichtwand. Auch hier wurden in einer Sonderkonstruktion die beiden Anforderungen Licht und Akustik kombiniert. Die LED-hinterleuchteten 
Wandelemente wurden mit einer Stoffbespannung versehen, die zusammen mit den an den Rückwänden angebrachten Absorbern für erstklassige akustische Werte sorgt. Das Licht, das sanft durch den Stoff scheint, und dessen weiche Materialität sorgen für eine Behaglichkeit und eine Atmosphäre, die im Kontext der ansonsten eher rauen und groben Ästhetik des Bestandsgebäudes etwas Besonderes ist.

Die Lichtwand steht vor den dominierenden Betonstützen und verdeckt diese, wodurch sich die Raumgrenzen in der Weite des Lichts auflösen - vielleicht in Analogie zur Unendlichkeit des Geistes und zum Ort der Bibliothek selbst, an dem Wissen sich bündelt und fortsetzt scheinbar bis ins Unendliche. Der auf den Stoff applizierte Motivdruck kann diese Assoziation noch verstärken.

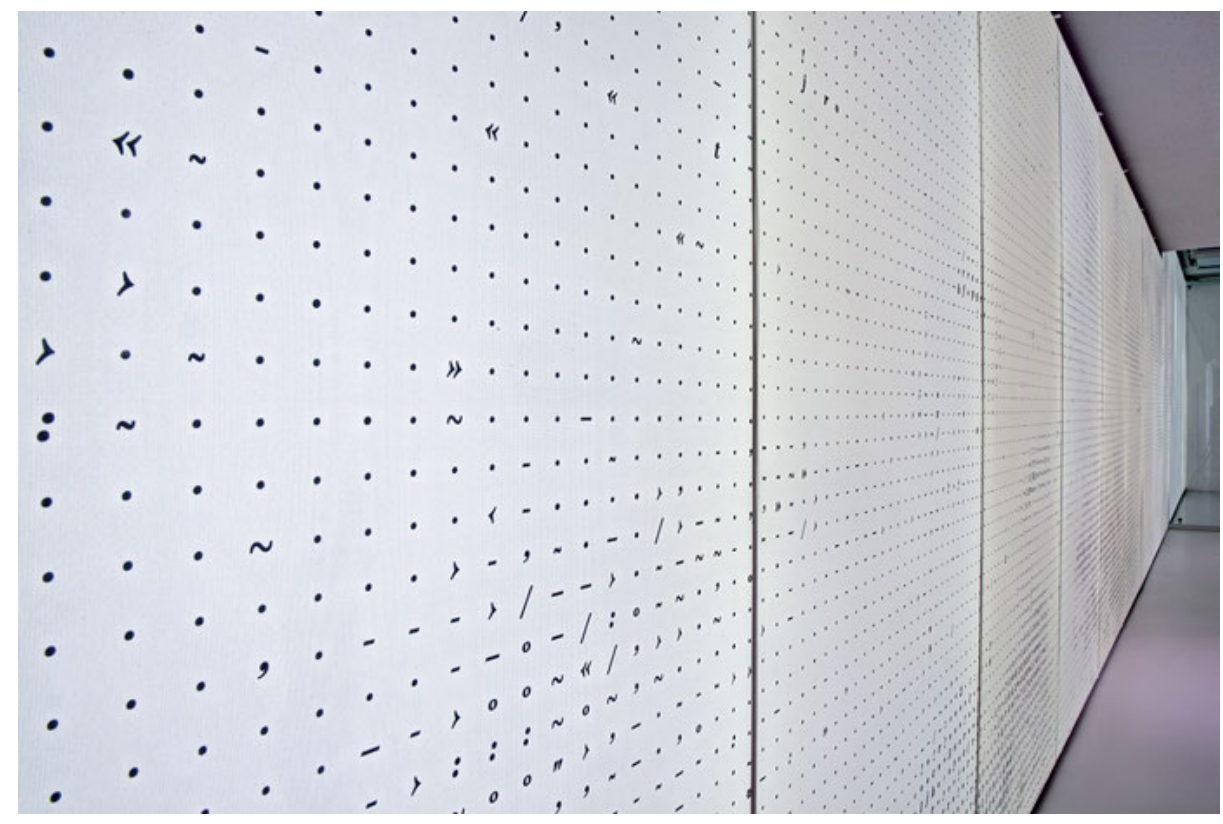

Abb. 16: Licht-Akustik-Wand im Informationszentrum (c) I. Reiter.

\subsubsection{Wand - Farbige Glastafeln}

Die Sonderräume im Informationszentrum und die Gruppenarbeitsräume im Verbindungsbau sind als Glasboxen ausgeführt. Jeweils ein Segment der Glaswände ist dabei als großformatige, gerahmte Farbfläche konzipiert. Jede Glasbox erhielt als Akzent eine der Spektralfarben und dadurch ihren Wiedererkennungswert. Mit dieser Gestaltungsmaßnahme wird das Statische des ursprünglichen Farbkonzepts, in dem einzelne Bauteile farbig angestrichen wurden, bewusst verlassen. Während man sich durch den Raum bewegt und dabei durch eine der Glasscheiben schaut, wird der 
Raum dahinter für einen Moment quasi eingefärbt. Dadurch entsteht für die Nutzer ein immer wieder neues Raum- und Farberlebnis. Auch bei diesem prägnanten Ausführungsdetail ist somit bewusst ein Bezug zu künstlerischen Strömungen der konzeptionellen Entstehungszeit der Hochschule in den 1960er Jahren hergestellt, die in die heutige Zeit hineinwirken. ${ }^{7}$

\section{Fazit}

Der gestalterisch-konzeptionelle rote Faden, der sich durch die Neugestaltung der Universitätsbibliothek Konstanz zieht, nimmt einige der 1960er-Jahre-Konzepte von Form, Farbe und Licht auf und überführt sie in eine zeitgemäße Architektur- und Materialsprache. Ziel war es, durch gezielte Farb- und Materialakzente eine klare und nutzerfreundliche Ausrichtung der Gestaltung zu schaffen.

In Anlehnung an die Urkonzeption und den Zeitgeist der Universitätsgründung führt die Neugestaltung das architektonische Spiel von Regel und Ausnahme, von Klarheit und Mystik, von Freiheit und Restriktion fort - Kontraste, die in der Tradition der klassischen Moderne stehen. Hier ging es in allererster Linie darum, die vorhandenen Qualitäten des Gebäudes aufzugreifen und durch die neuen Maßnahmen noch zu verstärken. Das ist eine Form des Respekts und der Wertschätzung im Umgang mit dem herausragenden ,Gesamtkunstwerk‘ der Universität Konstanz, wo sich Inhalte und Form im Sinne des Modernen und Zukunftweisenden zu einem Ganzen verbinden.

\section{Literatur und Internetquellen}

Frank, M. B. (2011). Eine Tonnenhalle von schlichter, puristischer Form und Räumlichkeit ... Die Stadtbücherei Mössingen zieht in ein denkmalgeschütztes spätmodernes Industriegebäude. In P. Hauke \& K. U. Werner (Hrsg.), Secondhand - aber exzellent! Bauen im Bestand (S. 238-253). Bad Honnef: Bock + Herchen. http://edoc.hu-berlin.de/miscellanies/secondhand-38495/238/ PDF/238.pdf (17.10.2015).

Naumann, U. (2009). Grundsätze des Bibliotheksbaus: Von den „Zehn Geboten“ von Harry FaulknerBrown zu den „Top Ten Qualities“ von Andrew McDonald. In P. Hauke \& K. U. Werner (Hrsg.), Bibliotheken bauen und ausstatten (S. 14-37). Bad Honnef: Bock + Herchen. http://edoc. hu-berlin.de/miscellanies/bibliotheksbau/ (17.10.2015).

Henning, W. (2011). „Flanieren Sie bitte!“ Ein bibliothekarischer Selbstversuch in der Mössinger Tonnenhalle. In P. Hauke \& K. U. Werner (Hrsg.), Secondhand - aber exzellent! Bibliotheken bauen im Bestand (S. 226-231). Bad Honnef: Bock + Herchen. Preprint: http://edoc.hu-berlin. de/miscellanies/secondhand-38495/238/PDF/238.pdf (19.10.2015).

7 Als wichtige Vertreter sind hier Donald Judd (Minimal Art) sowie Barnett Newman und Mark Rothko (Farbfeldmalerei) zu nennen. 